\title{
VALORACIÓN NUTRICIONAL DEL MENOR DE CINCO AÑOS Y COMPRENSIÓN DE INDICACIONES DEL CUIDADOR QUE ASISTE A CONSULTA CON PROFESIONAL ENTRENADO EN AIEPI
}

\section{NUTRICIONAL VALUATION OF THE MINOR OF FIVE YEARS AND UNDERSTANDING OF INDICATIONS BY CARETAKER WHO ATTENDS CONSULTATION WITH PROFESSIONALS TRAINED IN IMCI}

Luz Dalila Vargas Cruz ${ }^{1}$ Dora Stella Melo ${ }^{2}$

\section{RESUMEN}

En los países en desarrollo, la desnutrición es un problema de salud importante en la niñez. Aunque, no es registrada como causa básica de mortalidad, una proporción de las muertes menores de cinco años tienen desnutrición, como origen asociado. La valoración de los parámetros antropométricos y la responsabilidad del personal de salud frente a la ejecución y la gestión de los programas de promoción y de prevención son factores importantes para analizar. Esta investigación, se realizó para conocer la influencia de la estrategia de la Atención Integrada a las Enfermedades Prevalentes de la Infancia (AIEPI) en el registro y clasificación del estado nutricional y en la comprensión de los cuidadores sobre los tratamientos e indicaciones alimentarios, de consulta de control de crecimiento y desarrollo, de signos de peligro y de consulta de seguimiento. Se utilizaron los instrumentos propuestos por la estrategia y se realizó observación a la consulta y verificación al

${ }^{1}$ Enfermera M.Sc. Docente Facultad Enfermería, Universidad de Ciencias Aplicadas y Ambientales U.D.C.A Dirección para Correspondencia: Calle 222 No.55-37, Bogotá, D.C. luvargas@udca.edu.co

2 Enfermera M.Sc. Docente Facultad Enfermería, Universidad de Ciencias Aplicadas y Ambientales U.D.C.A Dirección para Correspondencia: Calle 222 No.55-37. cuidador de la información suministrada por profesional capacitado en estrategia y no capacitado. Los resultados indican que en la consulta con profesional capacitado en la estrategia AIEPI al 31,8\% se le realizó clasificación nutricional y en la consulta sin capacitación al $13,8 \%$. El $63 \%$ de los cuidadores que asistieron a la consulta AIEPI y el 58,6\% sin AIEPI mencionaron correctamente las indicaciones sobre cuándo venir en busca de ayuda. Se concluye que existen dificultades para la comprensión de las indicaciones dadas, relacionado posiblemente con el momento de la aplicación del estudio cuando la estrategia se estaba consolidando.

Palabras clave: AIEPI, nutrición, cuidadores, comprensión.

\section{SUMMARY}

In the developing countries, undernourishment is an important childhood health problem. Although, it is not registered as a basic cause of mortality, a death quota of children, younger than five years has undernourishment as an associate cause. The indices of malnutrition, the valuation of the anthropometric parameters and the responsibility of the health personnel regarding execution and management of promotion and prevention programs are important factors to analyze. This research was realized to know the effect of the Integrated Attention the Prevalent Illness of Children (IMCI) strategy in the 
registration and classification of the nutritional state and the understanding of caretakers of the treatment and the nourishing indications given, during consultation of Control of Growth and Development, signs of danger and consultation of pursuit. The instruments proposed by the strategy were used and observations of the consultation and a verification of the execution by the caretaker of the information given by trained and not trained professionals were made. The results indicate that in consultation with a trained professional for a $31,8 \%$, a nutritional classification was executed and in the consultation with not qualified personnel it corresponded to $13,8 \%$. $63 \%$ of the caretakers who attended the IMCI consultation and $58.6 \%$ without, mentioned correctly the indications when to come in search of help. The existence of difficulties in the comprehension of instructions was concluded, being related to the moment of the application of the strategy, since at the time of the research very few professionals had been trained.

Key words: IMCI, nutrition, caretakers, understanding

\section{INTRODUCCIÓN}

La Organización Panamericana de la Salud, la Organización Mundial de la Salud y el Fondo de las Naciones Unidas para la Infancia presentaron en 1996 la estrategia Atención Integrada a las Enfermedades Prevalentes de la Infancia, AIEPI. Desde entonces fue adoptada por la mayoría de los países en desarrollo, desencadenándose un rápido proceso de aplicación e incorporación de la misma como política (OPS, 1998). En Colombia, la estrategia fue adoptada de manera oficial por el Ministerio de Salud en 1998, iniciando los procesos de capacitación, con los ajustes acordes al perfil epidemiológico y a las necesidades en salud infantil de la población.

Si bien la desnutrición no es registrada como causa básica de mortalidad, la OPS (2001) afirma que una proporción variable de las muertes de niños menores de cinco años presentan desnutrición, como causa asociada.

La situación de desnutrición muestra una pequeña disminución, según la Encuesta Nacional de Demografía (Profamilia et al. 2000). Para el año 2000, el 14\% de los menores de cinco años sufrían de desnutrición crónica o retardo en el crecimiento; el 3\% de ellos alcanzaron grados de desnutrición severa, el $1 \%$ alcanzó grados de desnutrición aguda, convirtiéndose en la de menor prevalencia para el país. Profamilia et al. (2005) muestran que los índices de malnutrición en el país disminuyeron a $12 \%$ de desnutrición crónica: $10 \%$ con un grado de moderada y $2 \%$ de severa; este porcentaje es preocupante para los habitantes del Distrito Capital y para la zona del Atlántico que corresponden a las regiones de Colombia con mayor prevalencia de desnutrición crónica (Profamilia et al. 2005).

La situación actual de la desnutrición en Bogotá D.C., según la Alcaldía Mayor, Sistema de Vigilancia Alimentario y Nutricional - SISVAN (2005) es del 13,4\% para desnutrición crónica y de 5,3\% para desnutrición aguda, datos por encima de los registros nacionales. Esto, frente a la problemática de sobre peso y obesidad para la población infantil de $2,7 \%$ y $1 \%$, respectivamente. Lo anterior nos lleva a inferir que los índices de malnutrición crónica en Colombia, especialmente en el Distrito Capital, la valoración inadecuada de los parámetros antropométricos y la responsabilidad del personal de salud frente a la ejecución y gestión de los programas de promoción y de prevención son factores importantes para analizar. Los resultados de la presente investigación responden a esta inquietud: cंcuál es el efecto de la aplicación de la estrategia AIEPI en la valoración del estado nutricional y la comprensión de los tratamientos indicados a la madre o cuidador en la consulta al niño y niña de dos meses a cuatro años, atendidos en dos centros de salud del Hospital de Usaquén adscrito a la Secretaría Distrital de Salud de Bogotá?

La aplicación de la estrategia AIEPI en un servicio de salud significa que los niños y niñas que concurren a ese servicio de salud son sistemáticamente pesados y medidos, datos que son utilizados para clasificar el estado nutricional con base en la ubicación del peso y talla del niño en la curva de peso para la edad, la talla para la edad y peso para la talla. Durante la consulta, el profesional de salud debe explicar al cuidador las recomendaciones con relación al estado de salud, tratamiento, signos generales de peligro y consulta de seguimiento. El presente estudio tuvo como objetivo identificar la influencia de la estrategia AIEPI en la clasificación y valoración nutricional del menor de cinco años y en la comprensión de las indicaciones dadas al cuidador que asisten a consulta con profesional entrenado en la estrategia. 
Frente a la comprensión de los cuidadores que asisten a la consulta de profesional entrenado en la estrategia, un estudio de Zamora et al. (2002) en Bolivia reportó que el $49 \%$ de las cuidadoras, cuyos hijos tenían prescripción de medicamentos orales, recordaba cómo dar el tratamiento correctamente (nombre del medicamento, dosis por vez, número de veces por día y duración del tratamiento). En lo que se refiere a los indicadores suplementarios de comunicación interpersonal, la proporción de madres o cuidadoras de niño o niñas que recibieron consejería sobre tratamiento en general no supera la mitad. Igualmente, las cuidadoras que conocían tres aspectos de manejo de casos en el hogar fueron 32\%, en cambio sus respuestas sobre si pueden describir al menos dos signos para búsqueda de ayuda inmediata alcanzan el $65 \%$, valor probablemente influenciado por su experiencia previa y no precisamente por la consulta recién pasada.

Estos resultados son similares a los obtenidos por López et al. (2004) en México, quienes encontraron un contraste de $25 \%$ de madres capacitadas, con una evaluación previa del impacto de AIEPI determinando que $58 \%$ de las madres tenía una adecuada capacitación en atención del niño o niña enfermo en el hogar. Sugieren, los mismos autores, la necesidad de crear procesos educativos que promuevan la comprensión de su situación de salud, así como el desarrollo de actitudes, habilidades y destrezas y que además sean culturalmente aceptados por la comunidad, de probada efectividad, económicamente viables y enfocados a los comportamientos necesarios de ser cambiados en la población blanco.

Una encuesta realizada por Dorado (2000) para conocer la atención medica brindada a los niños menores de cinco años en instituciones de salud de primer nivel, encontró frente a la valoración nutricional que el $76 \%$ de los médicos pesaron a los niños y niñas; de éstos, el $19 \%$ presentó problemas de peso, pero solo al $43 \%$ le proporcionaron recomendaciones alimenticias. El estudio reporta que al $58 \%$ de los niños de 13 a 24 meses y al $40 \%$ de otras edades con problemas de peso no se dieron recomendaciones a los padres o acudientes sobre la adecuada alimentación. Alves Da Cunha et al. (2005) encontraron que la adherencia a la carta recordativa (la tarjeta, herramienta que se entrega a la madre o cuidador para recordarle cuándo volver de inmediato, cómo dar líquidos y recomendaciones para el niño sano y enfermo) fue solo del $50 \%$. Concluyen que el entrenamiento en los signos generales de peligro y cuándo volver debe ser reforzado.

López et al. (2004) indican que frente a la valoración nutricional al $66,9 \%$, se le ordenó o suplementó con vitamina A; al $50 \%$, se le realizó vigilancia nutricional y $25,5 \%$, se le capacitó a la madre y al cuidador. Concluyeron que los componentes de la AIEPI de la Infancia muestran coberturas acordes con su factibilidad y tiempo de implantación. Los hallazgos de la asociación de la atención integrada de la salud infantil destacan la importancia de reunir mayor evidencia sobre su efecto en el estado nutricional, con el fin de contribuir en el mejoramiento y de prevención de la desnutrición infantil.

\section{MATERIALES Y MÉTODOS}

Esta investigación fue de tipo descriptivo longitudinal, debido a que se tomaron los datos obtenidos de la observación de la consulta y de preguntas de verificación de comprensión al cuidador al salir de la consulta en dos centros de salud del Hospital de Usaquén, adscrito a la Secretaría Distrital de Salud de Bogotá, en el CAMI de Verbenal (profesionales entrenados en la estrategia AIEPI), con una muestra de 44 cuidadores y la UPA de San Cristóbal (profesionales no entrenados en la estrategia AIEPI), con una muestra de 29 cuidadores. Para determinar la muestra, se realizó una revisión de los niños atendidos durante los últimos seis meses en cada sitio y, se sometió los datos al método estadístico; sin embargo, cuando se estaba en la recolección en San Cristóbal se requería profesionales no capacitados y para diciembre de 2004 todos los profesionales entraron a capacitación, por lo cual, no se pudo llegar a 44 niños. Los criterios para la inclusión de la muestra fueron niños y niñas entre los dos meses y cinco años y sus cuidadores que asistieron a consulta con médico o enfermera.

Las Unidades Primarias Zonales (UPZ) utilizadas fueron Verbenal y San Cristóbal. La UPZ Verbenal, se localiza en el norte de la localidad de Usaquén y la de San Cristóbal Norte, en el área central. La población de menores de cinco años corresponde a 2,04\% niños menores de un año y el 7,85\% a niños entre uno y cuatro años (Alcaldía Mayor de Bogotá, 2004). 
Los instrumentos utilizados para la recolección de la información fueron los proporcionados por la estrategia para la observación de la consulta y para la medición de comprensión de indicaciones. Las consultas tomadas en cuenta dentro del estudio fueron las de crecimiento y desarrollo realizadas por médico y enfermera y la consulta al médico por algún evento de enfermedad.

Se plantearon las hipótesis de acuerdo a la variable de valoración del estado nutricional y comprensión de los tratamientos de Hipótesis Alterna: la aplicación de la estrategia AIEPI contribuye a mejorar los registros de peso, talla y clasificación del estado nutricional, así como la comprensión de los tratamientos indicados por el personal de salud en la madre o cuidador de los niños y niñas de dos meses a cuatro años, atendidos en dos centros de salud del Hospital de Usaquén. Hipótesis Nula: la aplicación de la estrategia AIEPI no contribuye a mejorar los registros de peso, talla y clasificación del estado nutricional y a mejorar la comprensión de los tratamientos indicados por el personal de salud en la madre o cuidador de niños y niñas de la misma edad atendidos en los centros de salud referenciados.

El estadístico apropiado para dichas hipótesis fue:

$$
\mathrm{Z}=\frac{\mathrm{P} 1-\mathrm{P} 2}{\frac{\mathrm{P} 1(1-\mathrm{P} 1)}{\mathrm{n} 1}+\frac{\mathrm{P} 2(1-\mathrm{P} 2)}{\mathrm{n} 2}} .
$$

Si Z $\geq Z 1$ - se rechaza la hipótesis nula, es decir, Ho.

El procedimiento realizado para la recolección de la información incluyó observar y registrar si el profesional pesó, talló y clasificó el estado nutricional, si preguntó el motivo de consulta, si educó sobre la enfermedad del niño, si instruyó sobre los tratamientos, para qué y cómo dar el medicamento, si enseñó sobre signos generales de peligro, consulta de crecimiento y desarrollo y consulta de control. Posterior a la consulta, se interrogó al cuidador sobre lo ocurrido en la consulta para determinar su comprensión.

\section{RESULTADOS Y DISCUSIÓN}

De las 44 consultas que aplicaron estrategia AIEPI, 28 fueron realizadas por una enfermera y 16 por un médico. De 29 consultas que no aplicaban la estrategia AIEPI, 18 fueron realizadas por enfermera y 11 por médico.
De los niños y niñas atendidos en consulta AIEPI, el porcentaje observado por enfermera es más alto por corresponder a los niños que asisten a consulta de crecimiento y desarrollo (70,45\%); comportamiento similar a las consultas realizadas por profesional sin capacitación en la estrategia. Un menor porcentaje (30\%) es la consulta que realiza el médico frente a sintomatologías comunes en la infancia, no muestra diferencias en este aspecto entre los grupos de capacitados y no capacitados.

Se encontró que el mayor porcentaje de los niños atendidos en los dos grupos asistían a la consulta de crecimiento y desarrollo. Ésta se constituye en una herramienta clave en el sector salud, ya que abarca mayor población que la consulta de urgencias y/o de evento de enfermedad; corresponde a uno de los primeros momentos de intervención donde la importancia de detectar alteraciones tempranas en el niño repercute en la intervención oportuna y la prevención de posteriores secuelas. Es entonces allí donde, a través de una valoración adecuada, se pueden detectar tempranamente las alteraciones nutricionales, del desarrollo y otros que pueda presentar el niño, incluidas las del contexto social y psicológico. Se destaca también el valor de la consulta de crecimiento y desarrollo, como fuente primaria de información para los cuidadores de los niños. Aquí se inicia el entrenamiento para la prevención de enfermedades y para la práctica de actividades que permiten el adecuado crecimiento y desarrollo.

Cáceres et al. (2005), al describir la relación entre la infección del rotavirus, la deshidratación por diarrea en niños colombianos menores de cinco años y los factores de riesgo de la diarrea con deshidratación, encontraron dentro de las variables analizadas que la falta de conocimientos previos acerca de la diarrea y la deshidratación y el no asistir a la consulta de crecimiento y desarrollo mostraron una fuerte asociación con la deshidratación por diarrea. "El efecto protector que desempeña la asistencia de los niños a las consultas de control de crecimiento y desarrollo en los últimos meses $(P=0,606)$ indicaron que esta variable estaba asociada con una mejor evolución".

Valoración y clasificación nutricional: Al 100\% de los niños se les realizó medición de peso y talla, por lo tanto, todos los niños fueron valorados nutricionalmente 
en cuanto a medidas antropométricas y registrados debidamente en la Historia Clínica.

De los niños y niñas pesados y tallados en la consulta con estrategia AIEPI, al 31,8\% (14), se le realizó clasificación nutricional y, en la consulta sin estrategia AIEPI, al 13,8\% (4), utilizando los términos contemplados en la estrategia, como son: no tiene desnutrición, desnutrición o riesgo y desnutrición grave. Se observa una pequeña diferencia: los capacitados en la estrategia realizan en menor proporción mayor valoración y clasificación nutricional que los no entrenados, sin embargo, estadísticamente, en cuanto a la realización de clasificación nutricional, no se encontraron diferencias ( $p>0,05)$ entre las consultas de los niños con estrategia AIEPI y sin estrategia AIEPI, al igual que en el registro de clasificación nutricional. Ésta es importante en la consulta al menor, independiente del motivo de asistencia, por que establece su estado nutricional e identifica tempranamente factores de riesgo nutricionales que puedan exponer a otras patologías o afectar su crecimiento y desarrollo normal.

Es muy significativo valorar los cambios a lo largo del tiempo, ya que una medida aislada tiene poco valor. Las mediciones seriadas nos van a permitir: a) construir un perfil de desarrollo del niño y b) calcular su velocidad de crecimiento. Registrar los datos en las curvas de crecimiento y hacer el seguimiento longitudinal de cada niño permitirá mostrar cuál es su canal de crecimiento y detectar cuándo desvía su percentil habitual. Esto aporta una información valiosa para interpretar el crecimiento y el estado nutricional del niño (Martínez $\mathcal{E}$ Pedrón, 2002).

Además, es importante anotar que en las consultas, tanto con el médico como con la enfermera, el registro de medidas antropométricas se convierte en un elemento más de la valoración nutricional, el cual se debe complementar con el análisis de la situación particular del niño o niña, en aspectos como: estado socioeconómico y cultural, que determina el acceso y la disponibilidad hacia los tipos de nutrientes; el aspecto emocional y las relaciones familiares, que permiten la aceptación, la asimilación y la tolerancia a cierto tipo de alimentos; el nivel educativo y cognitivo del cuidador o del individuo, que estipula el grado de conocimiento sobre la importancia de la nutrición en el desarrollo del niño, así como la comprensión que da a las recomendaciones dadas en una determinada intervención.
Comprensión de las indicaciones dadas: La comprensión de los tratamientos fue evaluada con la aplicación de la encuesta, donde, el término correcto, se asigna cuando el cuidador mencionó completamente las indicaciones dadas por el personal de salud y el término incorrecto, se asigna cuando el cuidador no mencionó, mencionó errada o parcialmente las indicaciones dadas por el profesional de salud.

Al total de los cuidadores que asistieron a consulta, se les informó sobre la situación o diagnóstico del niño y se les dieron indicaciones pertinentes, pero un poco más de la mitad logró comprender el cien por ciento de la información suministrada, en los dos grupos, demostrando que por múltiples factores la comprensión de los usuarios, se ve comprometida.

En cuanto a comprensión de las indicaciones dadas sobre "le dijeron qué tenía el niño", no existió una diferencia estadísticamente significativa entre los dos grupos; sin embargo, se evidencia que un $8 \%$ más de los cuidadores que asistieron a consulta con estrategia AIEPI mencionaron correctamente lo que afecta al niño, frente al grupo de no AIEPI; se destaca, entonces, cómo el porcentaje para los dos grupos fue de apenas el $50 \%$, lo que manifiesta que faltó una mayor claridad para expresar y hacerse comprender frente a los cuidadores, como se observa en el gráfico 1.

Es importante recalcar que las respuestas dadas frente a lo que padecía el niño, se pueden ver influenciadas por las expectativas previas del cuidador a la consulta, de tal manera que éste no menciona la totalidad de las indicaciones dadas sobre el cuidado del menor, sino lo más significativo, según su percepción y sus expectativas. El 95,5\% de los padres con niños atendidos con estrategia AIEPI y el $100 \%$ de los atendidos sin estrategia AIEPI mencionan que se encuentran conformes con las indicaciones brindadas, por tanto, no existen diferencias entre una consulta y otra.

Influye también en la comprensión del cuidador la forma en que el profesional de salud aborda al paciente y la forma en que se hace entender; en esto influyen aspectos, como el número de pacientes asignados para consulta, el tiempo asignado para las consultas y la disponibilidad del profesional. 


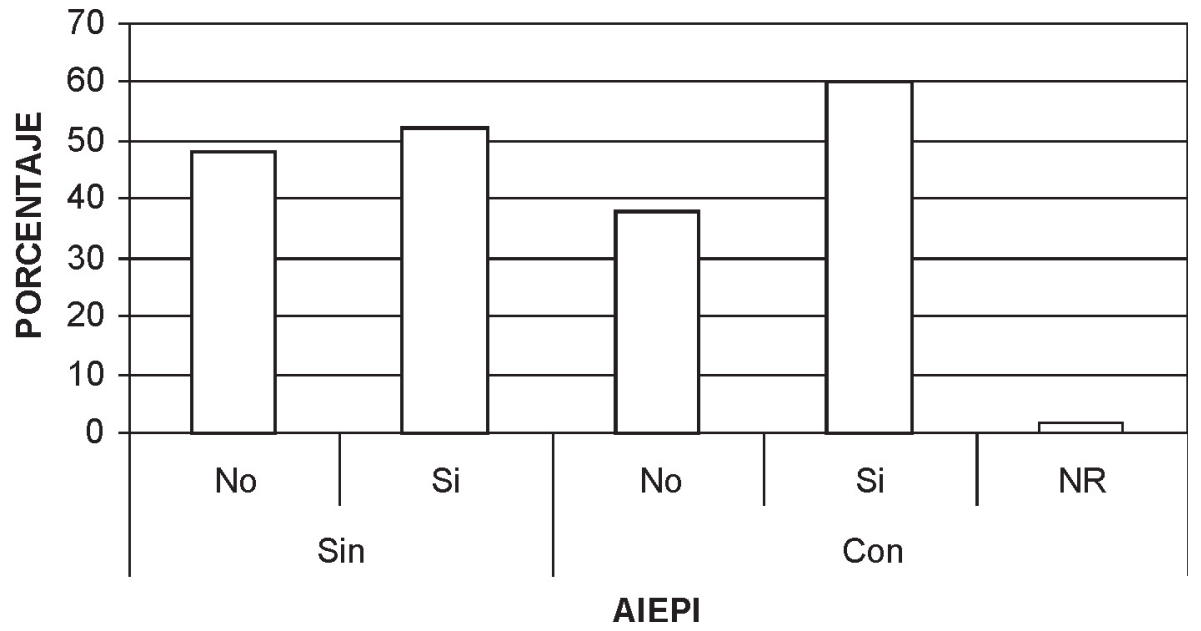

Gráfico 1. Comprensión de las indicaciones dadas en la consulta al menor de cinco años.

Ordenaron medicamentos: Al 45,5\% (20) de los niños atendidos por AIEPI y al 75,9\% (22) de los niños atendidos sin estrategia AIEPI, se les formuló medicamentos, por tanto, sí existen diferencias al formular medicamentos entre una consulta y otra. Lo anterior permite observar que la estrategia AIEPI aún sigue siendo la estrategia ideal para el uso racional de los medicamentos (gráfico 2). El 50\% de los medicamentos 1 y 2 formulados en la consulta atendida por AIEPI y el $9 \%$ de los medicamentos 1 y 2 formulados en la consulta atendida sin AIEPI fueron entregados al salir el paciente de la consulta, por tanto, sí existen diferencias en la entrega del medicamento al paciente.

En cuanto al suministro de la primera dosis de los medicamentos, no existieron diferencias entre los dos grupos. Aunque en la estrategia AIEPI se entregan los medicamentos, pero no se suministran, debido a la situación del menor y a los mecanismos de repartición de los mismos, ya que no eran suministrados por el profesional de la consulta sino por el farmaceuta, por lo tanto, la estrategia se había implementado parcialmente. Actualmente, en algunos Centros de Atención, hay una auxiliar de enfermería quien asume esta labor.

En cuanto al medicamento formulado en la consulta con estrategia AIEPI, el 57\% fue entregado y en uno de los casos fue suministrado inmediatamente al menor, por las características de salud del menor (fiebre). En el 100\% de los formulados en la consulta sin AIEPI, no se registró si fueron entregados y suministrados, debido a los

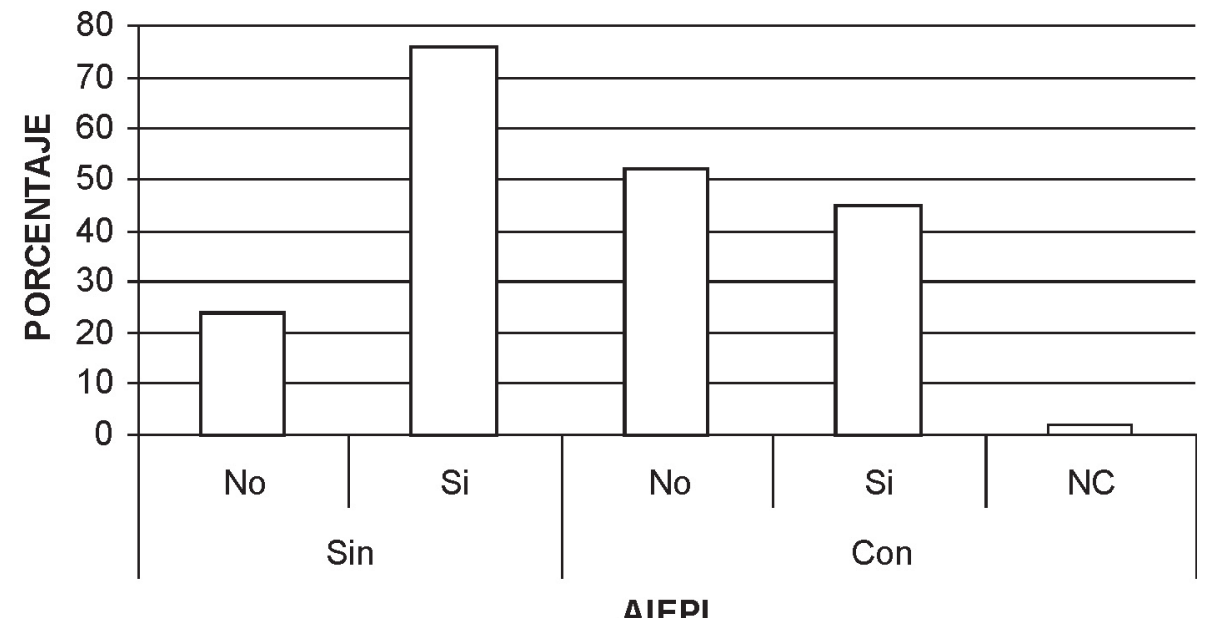

Gráfico 2. Formulación de medicamentos en la consulta al menor de cinco años. 
mecanismos de entrega que dificultaron el seguimiento. El 100\% (42) de las madres o cuidadores de los niños a quienes se les formuló algún fármaco mencionaron correctamente que medicamento tenía que darle al niño tanto en los que aplicaron AIEPI como los que no, por tanto, no existen diferencias entre los grupos.

Para qué sirve cada medicamento: De los cuidadores de los niños atendidos por profesional capacitado en AIEPI y que les formularon medicamentos (20), el $20 \%$ (4) de los cuidadores manifestaron que les indicaron para qué servía; y de los 22 niños sin estrategia AIEPI, también formulados, el 72,7\% (16) de los cuidadores declararon que les dijeron para qué servía cada medicamento. En este caso existen diferencias al mencionar que sí les dijeron para qué era útil. Es importante señalar que en algunos pacientes de la estrategia AIEPI ya conocían el medicamento ordenado. El $20 \%$ de las madres de los niños con estrategia AIEPI a quienes formularon medicamentos indicaron correctamente para qué sirven y el $41 \%$ de las madres de los niños sin estrategia AIEPI mencionaron acertadamente para qué se utilizan los medicamentos. Aunque se observa divergencias en los grupos, estadísticamente, el valor $\mathrm{p}<0,05$ muestra que no existen diferencias significativas.

Como se observa en los resultados, los porcentajes de comprensión de para qué servían los medicamentos para los dos grupos fueron menores del $60 \%$, lo que indica que existió dificultad en la percepción total de las indicaciones dadas en cuanto a entendimiento sobre para qué se ordena el medicamento a los niños.

Cómo dar cada medicamento (cantidad, cuántas veces, cómo, cuántos días): De los cuidadores de los niños que les formularon medicamentos, el $90 \%$ de los atendidos por AIEPI y el 95,5\% de los atendidos sin AIEPI, les informaron cómo dar cada medicamento y 95\% de los atendidos por AIEPI y sin AIEPI manifestaron correctamente que les dijeron cómo suministrarlos, por tanto, no existen diferencias. El 35\% de las madres de los niños con estrategia AIEPI y el $32 \%$ de las madres de los niños sin estrategia AIEPI mencionan acertadamente cómo tienen que darle el medicamento al niño. Aunque se observan diferencias menores entre los grupos, estadísticamente el valor $\mathrm{p}<0,05$ muestra que no existen diferencias en los grupos.

El 70\% de las madres de los niños con estrategia AIEPI y el $59 \%$ de las sin estrategia AIEPI explican correctamente por cuántos días tiene que darle el medicamento. Estadísticamente, el valor $\mathrm{p}<0,05$, muestra la ausencia de diferencias. A pesar de esto, se observan (gráfico 3) diferencias entre los dos grupos: la comprensión de cómo administrar los medicamentos y durante cuántos días es mayor en la consulta de los capacitados en AIEPI, y la comprensión de para qué sirve, cuánto y cuántas veces es mayor para los no capacitados en la

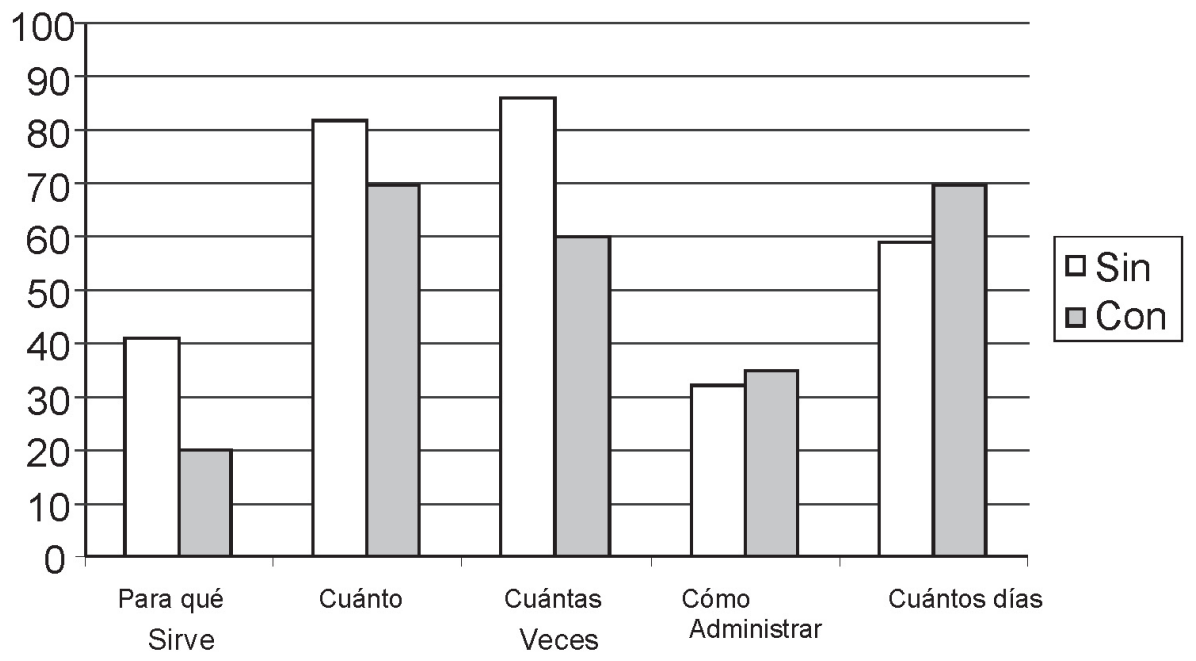

Gráfico 3. Comprensión de la administración de medicamentos en la consulta del menor de cinco años. 
estrategia. Una de las razones que pudo originar estos resultados puede ser que en los no capacitados la consulta la dirigen hacia las necesidades del niño y su cuidador; mientras en los capacitados pueden dirigir la consulta a involucrar todos los aspectos relacionados a la situación integral del menor, lo que puede producir que, al final de la consulta, la madre o cuidador no recuerde completamente las indicaciones sobre la administración de los medicamentos.

Signos generales de peligro: No se encontraron diferencias en los dos grupos, pero se observa un porcentaje alrededor del $40 \%$ para los que mencionan correctamente las indicaciones dadas, lo que muestra, como se mencionó anteriormente, que existen dificultades para la comprensión total de los medicamentos y de las indicaciones dadas. Es importante, en este sentido, realizar mayor capacitación a los profesionales de salud para realizar mejor la educación sobre los tratamientos, así como el seguimiento al cumplimiento de dichas recomendaciones.

Control de crecimiento y desarrollo y sobre la consulta en general: Los resultados indican que los dos grupos mencionan a la madre cuándo volver a control de la situación que motivó la asistencia médica. En la consulta de crecimiento y desarrollo denota diferencias, encontrando que se explica más sobre la consulta de crecimiento y desarrollo en los profesionales que no han sido capacitados. Como en lo anteriores aspectos, en la información que se suministra sobre la consulta de control y la consulta de crecimiento y desarrollo, denota una baja comprensión de la información suministrada. Sumado a las inferencias realizadas anteriormente, se considera que no se hace valoración de la comprensión suministrada, es decir, el profesional no verifica que la información haya sido comprendida en su totalidad.

Sobre la Consulta en General: Para los dos grupos, los cuidadores no refieren inconvenientes importantes que no permitan la asistencia a las citas de control o de crecimiento y desarrollo.

\section{CONCLUSIONES}

La estrategia AIEPI no tuvo un efecto significativo en los profesionales capacitados en el centro de salud en el registro de la valoración del estado nutricional de los niños, sin embargo, se mejoró el registro de la clasificación nutricional cuantitativamente, es decir se pesa y se talla al niño, se registran estos datos pero no se da una valoración cualitativa de esto valores dejando, de esta manera, inconcluso el proceso. Esto se relaciona también con el momento en que se realizó el estudio, ya que estaba iniciado el proceso de capacitación pero no estaban bien consolidados los procesos de evaluación y de seguimiento a los capacitados.

Es significativo priorizar la información que se brinda al cuidador, de acuerdo a la situación que presenta el niño, a los riesgos que incurre para su grupo de edad y a las expectativas del cuidador, involucrando los elementos de la comunicación, que la estrategia propone, para que la relación terapéutica que se cree con el usuario y el respeto por su cosmovisión y creencias de la salud sea un elemento importante para lograr la construcción de actitudes que generen comprensión y, por supuesto, cambio.

\section{RECOMENDACIONES}

Realizar investigaciones después de consolidados los procesos de capacitación y de evaluación que permitan determinar la comprensión de las indicaciones y los tratamientos de los cuidadores de niños y niñas y su adherencia y seguimiento en el hogar, así como los factores culturales, sociales, económicos y otros que intervienen, para que ocurra o no el cumplimiento a las recomendaciones.

\section{AGRADECIMIENTOS}

A Esperanza Avellaneda por la asesoría constante y su apoyo para realizar la investigación.

\section{BIBLIOGRAFÍA}

ALCALDIA MAYOR DE BOGOTÁ, SISTEMA DE VIGILANCIA EPIDEMIOLOGICA ALIMENTARIA Y NUTRICIONAL SISVAN. 2005. Comportamiento del estado nutricional por grupo etareo según el indicador talla para la edad en menores de 7 años notificados en el SISVAN, enero a Junio del 2005. Disponible desde Internet en: http://www. saludcapital.gov.co/secsalud/boletines_epidemiologicos/Boletin0605_archivos/Page1038.htm (con acceso en 03/08/07.) 
ALCALDÍA MAYOR DE BOGOTÁ. 2004. Secretaría de Hacienda, Departamento de Planeación, Recorriendo a Usaquén, Diagnóstico Físico y socioeconómico de las localidades de Bogotá, 2004. Disponible desde Internet en: http://www.dapd.gov. co/www/resources/tqv_recorriendo_usaquen.pdf (con acceso 03/06/07).

ALVES DA CUNHA, A.J.L., DOS SANTOS, S.R., MARTINES, J. 2005. Integrated care of childhood disease in Brazil: Mothers' response to the recommendations of health workers. Acta Paediatrica Promoting Child Health, 94(8):1116-1121.

ASOC. PROBIENESTAR DE LA FAMILIA COLOMBIANA (PROFAMILIA), INST. COL. BIENESTAR FAMILIAR (ICBF); MIN. SALUD; FUNDACION CORONA; CONSEJERIA PRESIDENCIAL PARA LA EQUIDAD DE LA MUJER; USAID; UNICEF; MEASURE/DHS, MACRO INTERNACIONAL Inc.; FED. INTERNAL PLANIFICACION FAMILIAR (IPPF-RHO). Salud Sexual y Reproductiva, Resultados Encuesta Nacional de Demografía y Salud 2000, Capitulo X: Lactancia y Nutrición de Niños y Madres, 2000, p. 146. Disponible desde Internet en: http://www.profamilia.org.co/encuestas/01encuestas/pdf_2005/ capitulo_XI.pdf (con acceso 03/08/07).

ASOC. PROBIENESTAR DE LA FAMILIA COLOMBIANA (PROFAMILIA), INST. COL. DE BIENESTAR FAMILIAR (ICBF); MIN. PROTECCIÓN SOCIAL; USAID; UNFPA. Salud Sexual y Reproductiva, Resultados Encuesta Nacional de Demografía y Salud 2005, Capitulo XI: Lactancia y Estado Nutricional, 2005, p.267. Disponible desde Internet en: http://www. profamilia.org.co/encuestas/01 encuestas/pdf_ 2005/capitulo_XI.pdf (con acceso 03/08/07).
DORADO P., E.A. 2000 Análisis de los resultados de la encuesta realizada en Medellín Bolívar, Turbo, Barranquilla, Sabana Larga, Santa Fe de Bogotá, Bogotá. Documento de la Organización Panamericana de la Salud. 55p.

LÓPEZ F., F.; BARQUERA C., S.; KAGEYAMA E., M. 2004. Evaluación de una atención integrada contra la desnutrición infantil en áreas urbanas de Nayarit, México. Salud pública Méx. 46(2):158-163.

MARTÍNEZ, C.C.; PEDRON, G.C. 2002. Valoración del estado nutricional, en Protocolos diagnósticos y terapéuticos en pediatría, p. 375, 377. Editorial AEP, España. Disponible desde Internet en: http:// www.aeped.es/protocolos/nutricion/9.pdf (con acceso 03/08/07).

ORGANIZACIÓN PANAMERICANA DE LA SALUD, OPS. 1998. Ministerio de Salud Pública del Ecuador. AIEPI en países de América Latina. Reunión de Evaluación y Planificación. Documento. QuitoEcuador. p.3.

ORGANIZACIÓN PANAMERICANA DE LA SALUD, OPS. 1999. Ministerio de Salud Boletín Noticias sobre AIEPI. Número 2. Diciembre 1999. p.1.

ORGANIZACIÓN PANAMERICANA DE LA SALUD, OPS. 2001. Investigaciones operativas sobre AIEPI. Abril de 2001. p.48.

ZAMORA, A.D.G.; CORDERO, D.V.; MEJIA S., M.2002. Evaluación de la estrategia "Atención integrada a las enfermedades Prevalentes de la infancia" (AIEPI) en Servicios de Salud, Bolivia. Rev. Chilena de Pediatría. 73(2):184-191.

Recibido: junio 6 de 2007

Aceptado: noviembre 9 de 2007 